\title{
The calibration and alignment of the LHCb RICH system
}

\author{
A. Papanestis \\ STFC—Rutherford Appleton Laboratory, OX11 0QX, UK
}

On behalf of the LHCb RICH Collaboration

\section{A R T I C L E I N F O}

Available online 19 July 2008

\section{Keywords:}

$\mathrm{LHCb}$

$\mathrm{RICH}$

Cherenkov

Alignment

Calibration

\begin{abstract}
A B S T R A C T
Hadron identification in the LHCb experiment is performed by two Ring Imaging Cherenkov (RICH) detectors, comprising three radiators and 484 Hybrid Photon Detectors (HPDs). A well calibrated and aligned RICH system is essential for providing the particle identification performance necessary for realising the physics goals. The total number of mirrors in the two RICH detectors is 116 and they must be aligned to an accuracy of $0.1 \mathrm{mrad}$ in order not to degrade the performance of the system. The refractive indices $n$ of the two gas radiators, namely $\mathrm{C}_{4} \mathrm{~F}_{10}$ and $\mathrm{CF}_{4}$, are expected to change with atmospheric pressure, and the refractivity $(n-1)$ will be monitored to an accuracy of $0.1 \%$ from physics data. The Cherenkov angle resolutions and photon yields will be calibrated using saturated Cherenkov rings and particles of known type. Both RICH detectors operate in the fringe field of the LHCb dipole magnet, which produces image distortions of the HPDs. Solutions to correct for these distortions are in the implementation phase. Finally, the overall performance of the RICH system for hadron identification will be evaluated using samples of pions and kaons identified from $\mathrm{D}^{*}$ decays, which can be isolated with high purity independently of the RICH itself.
\end{abstract}

(c) 2008 Elsevier B.V. All rights reserved.

\section{Introduction}

The purpose of the Ring Imaging Cherenkov (RICH) system [1] of the $\mathrm{LHCb}$ experiment $[2,3]$ is to provide hadron identification over the momentum range $1-100 \mathrm{GeV} / c$. This challenge has been Omet using two RICH detectors. RICH 1 is positioned close to the interaction point with its entrance window at a distance of $1.0 \mathrm{~m}$. It contains two radiators, $50 \mathrm{~mm}$ thick aerogel and $\sim 1 \mathrm{~m}$ of $\mathrm{C}_{4} \mathrm{~F}_{10}$, and provides particle identification over the range $\sim 1-65 \mathrm{GeV} / c$. ORICH 2 is positioned after the tracking stations with its entrance window at a distance of $9.5 \mathrm{~m}$ from the interaction point. It uses a $\mathrm{CF}_{4}$ gas radiator and provides particle identification over the range $\uparrow 25-100 \mathrm{GeV} / \mathrm{c}$ and beyond.

Both RICH detectors have similar optical geometry. A set of spherical mirrors focuses the Cherenkov light cone into a ring and a set of planar mirrors reflects these photons outside the acceptance of the experiment, where the photon detectors are placed. RICH 1 has vertical geometry, with the photon detectors above and below the beam pipe; RICH 2 has horizontal geometry with the photon detectors located on both sides. There are four spherical and 16 planar mirrors in RICH 1, and 56 spherical and 40 planar mirrors in RICH 2. The aim for the alignment of these mirrors is 0.1 mrad. Hybrid Photon Detectors (HPDs) [4] are used

E-mail address: a.papanestis@rl.ac.uk to detect the Cherenkov photons, 196 HPDs in RICH 1 and 288 in RICH 2. Although the HPDs are placed inside iron boxes to shield them from the fringe field of the LHCb magnet, image distortions are observed and must be corrected.

\section{Correction for magnetic field distortions}

The photon detectors of the two RICH detectors lie in the fringe field of the $\mathrm{LHCb} 4 \mathrm{Tm}$ dipole magnet, and experience up to a maximum of $2.4 \mathrm{mT}$ in regions of RICH 1 . Although the HPDs are locally shielded, electron trajectories inside the HPDs will be distorted with respect to magnetic field off [5]. Correction factors will need to be applied to every HPD in the longitudinal and transverse directions, and these corrections will be extracted by imaging test patterns onto the photo-cathodes of each HPD. The patterns will be compared with different LHCb magnet settings (field off and on, field reversed).

In $\mathrm{RICH} 2$ the test pattern will be projected using a commercially available overhead projector. The intensity of the projector is attenuated using neutral density filters to a few hits per HPD per $25 \mathrm{~ns}$ time window. A single spot from the projector, when projected $5 \mathrm{~m}$ to the HPD plane, creates 3-4 hits, each hit of size $2.5 \times 2.5 \mathrm{~mm}^{2}$. By using an appropriate clustering algorithm employing a centre of gravity method, the spot positions can be identified and fitted. Fig. 1 shows the three steps of the process. 


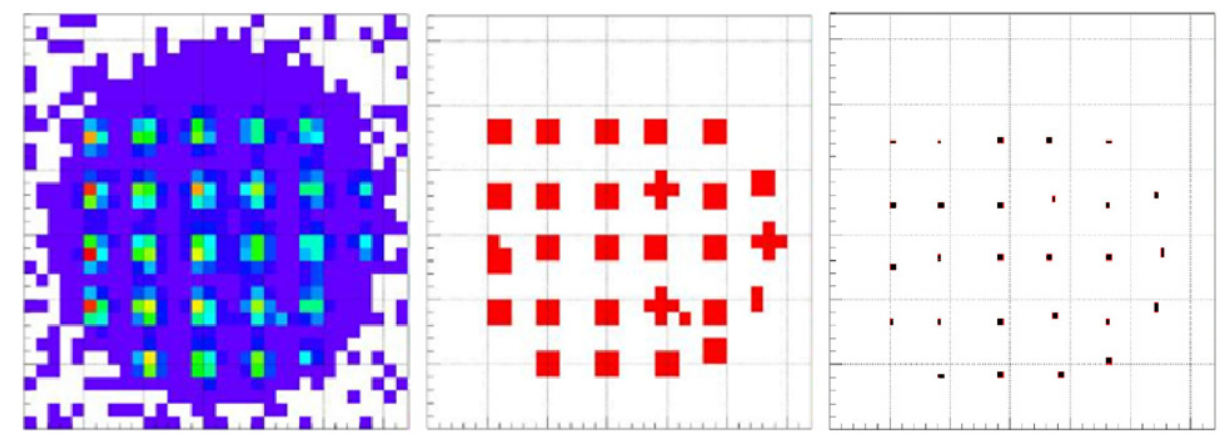

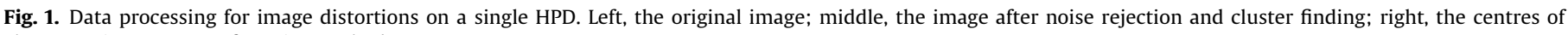
clusters using a centre of gravity method.

In the test set-up in the laboratory an accuracy of 0.5 pixels was achieved, which corresponds to $1.25 \mathrm{~mm}$ on the HPD photocathode. Surveyed photo-multipliers on the peripheries of the HPD arrays will allow accurate location of the projected pattern onto each HPD.

In RICH 1 a different method of magnetic calibration is required because of space restrictions. A linear array of precisely positioned and collimated LEDs mounted on an $x-y$ stage will be illuminated directly in front of the photon detectors and the full range of pixel positions scanned.

The same regular test patterns as used for the magnetic distortion measurements can also be used for HPD-to-HPD alignment. The centres of the identified clusters can be converted to space points in the LHCb coordinate system and these points can be used to define straight lines between the patterns across HPDs. Any HPD misalignment will appear as a deviation from a straight line and the residuals can be used to extract the position of each HPD.

\section{Alignment}

Once the LHCb experiment starts data-taking, it will be possible to align the RICH detectors using particle tracks, both internally (e.g. alignment of mirror segments) and with the rest of the experiment.

Any misalignment of the RICH detectors results in a dependence of the Cherenkov angle, $\theta_{\mathrm{ch}}$, on the measured azimuthal angle around the ring $\phi_{\mathrm{ch}}$, defined in Fig. 2. Without any misalignment, the projection of the track that emits the Cherenkov photons defines the centre of the ring shown by position $\mathrm{C}$. The radius of the ring relates to $\theta_{\mathrm{ch}}$, which should be constant for each photon measurement and independent of $\phi_{\mathrm{ch}}$. Any misalignment in the RICH components (e.g. the mirrors) moves the positions of the photons from those expected, but not the imaged position of the track. With the centre of the misaligned ring in position $\mathrm{C}^{\prime}$, the reconstructed Cherenkov angle becomes $\theta_{0}$, which now depends on the value of $\phi_{0}$. The Cherenkov angle of saturated particles $(\beta \approx 1)$ can be calculated, so it is possible to plot $\Delta \theta=\theta_{0}-\theta_{\text {expected }}$ as a function of $\phi_{0}$. This gives a cosine curve of the form

$\Delta \theta=A \cos \left(\phi_{0}\right)+B \sin \left(\phi_{0}\right)$

where $A$ and $B$ are misalignment coefficients. For the mirrors, $A$ and $B$ are the combined misalignments of the spherical and planar mirrors, so a further minimisation step is required to extract the parameters for individual mirror segments. This technique can be used to align the optical parts of the RICH system with an accuracy of $0.1 \mathrm{mrad}$ and is described in more detail in Refs. [6,7].

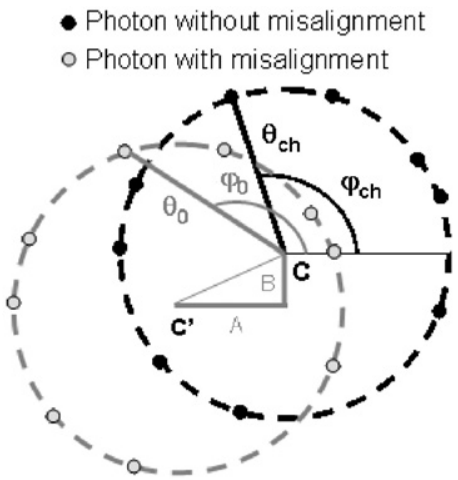

Fig. 2. Cherenkov photons distributed on a ring. The position $C$ marks the centre of the ring defined by the imaged track without any misalignment. Any misalignment in the optical system results in a movement of the photons, but not the imaged position of the track. The centre of the misaligned ring is in position $C^{\prime}$. $A$ and $B$ are defined in Eq. (1).

Fig. 3 shows alignment results from a test-beam study in September 2006 [8]. The set-up consisted of a Cherenkov vessel with $\sim 1.1 \mathrm{~m}$ of $\mathrm{C}_{4} \mathrm{~F}_{10}$ radiator with a beam of $80 \mathrm{GeV} / c$ negative pions. The Cherenkov ring was imaged by a parabolic mirror with $1.0 \mathrm{~m}$ focal length onto four close-packed HPDs. The positions of the HPDs are used, as well as the mirror orientation, to obtain the alignment for the full ring. Since the HPD positions are accurately known, the biggest contribution to misalignment comes from the uncertainties of the positions of the pixel sensors inside the different HPDs. These positions were measured independently during the HPD qualification process [9] and, when included in the geometrical description, lead to accurate alignment.

\section{Cherenkov angle performance}

The performance of the RICH detectors in reconstructing the Cherenkov angle can be evaluated from high momentum saturated tracks. Fig. 4 shows the distribution of reconstructed Cherenkov angles in RICH 2 for track momenta above $80 \mathrm{GeV} / c$. The data were produced with the LHCb Monte-Carlo simulation and contain at least one B meson in the event. The Cherenkov angle resolution of RICH 2 is expected to be $0.7 \mathrm{mrad}$, which has been evaluated using only photons associated with the correct track from Monte-Carlo truth information. In Fig. 4, since no Monte-Carlo truth information is used, a significant number of photons are reconstructed using the wrong track. Fitting the peak, a sigma of $0.8 \mathrm{mrad}$ is obtained, implying the distribution of background photons (wrong track association) under the peak slightly degrades the measurement. Studies are underway to 

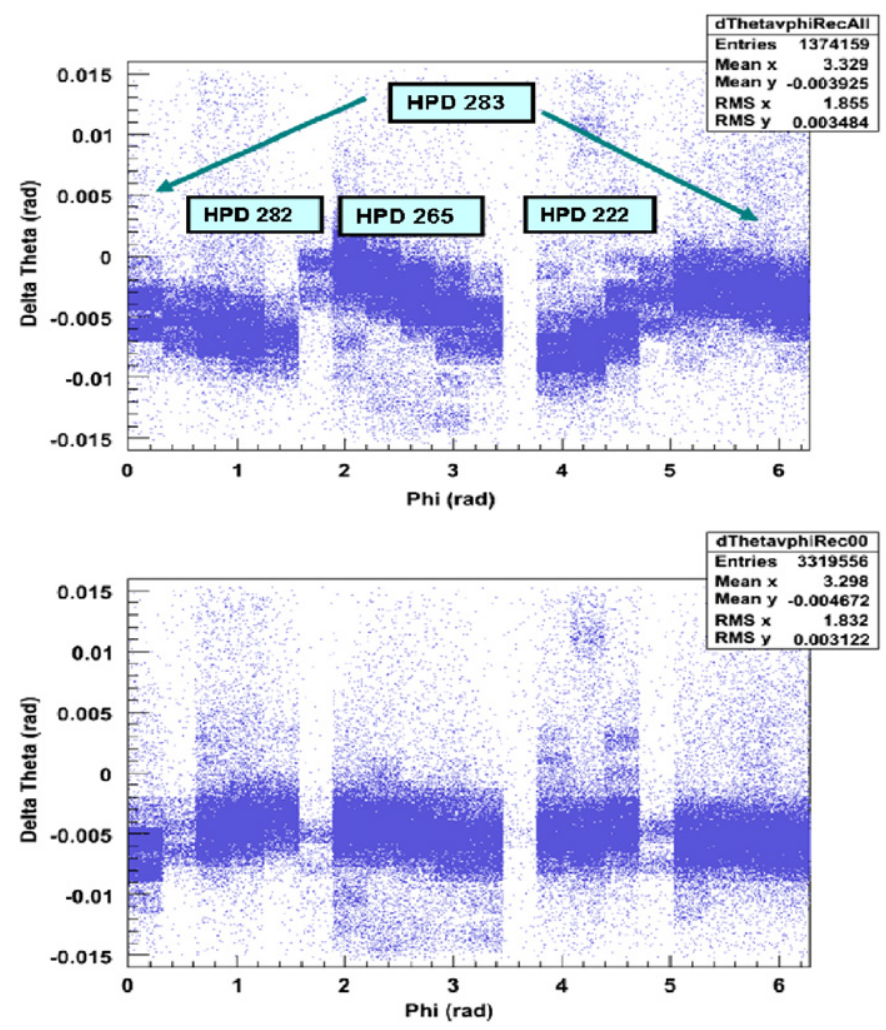

Fig. 3. Alignment histograms showing $\Delta \theta=\theta_{0}-\theta_{\text {expected }}$ as a function of $\phi_{0}$ from the September 2006 test-beam analysis. The boundaries of HPDs are indicated. The top histogram shows the alignment without correcting for the positions of the HPD pixel sensors. The bottom histogram shows the alignment parameters after the known positions of the pixel sensors are included in the geometrical description. The mean value of the vertical axis is not zero because the refractive index of the radiator had not yet been fully calibrated.

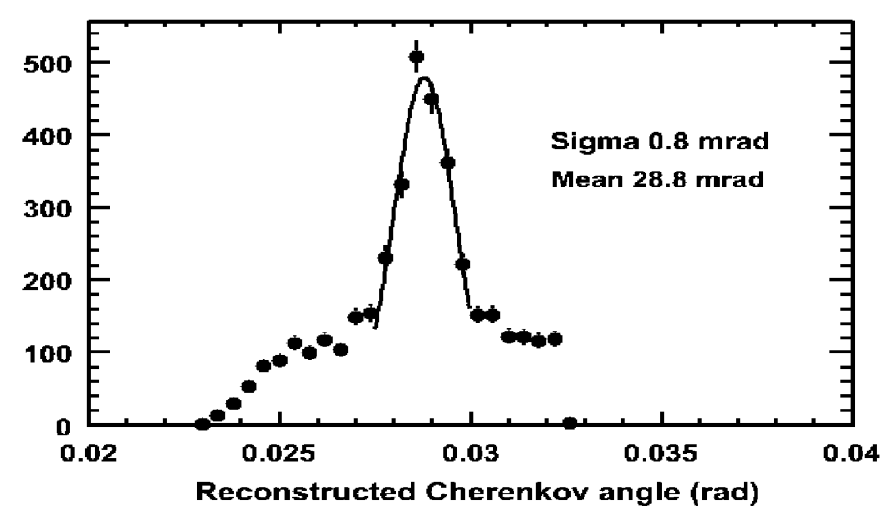

Fig. 4. Histogram showing the Cherenkov angle in RICH 2 determined from all photons from tracks with momenta above $80 \mathrm{GeV} / c$. The peak contains mainly photons associated with the correct track, while the broader part results from photons mainly mis-associated with the wrong track. The width of the peak gives the Cherenkov angle resolution, while the average refractive index, convoluted with the wavelength-dependent quantum efficiency of the photon detectors, can be extracted from the mean value of the peak.

identify isolated rings in order to reduce the number of background photons and improve the extracted resolution.

The mean value of the fit can be used to monitor changes in the refractive index of the gas radiator. The $\mathrm{RICH}$ gas radiators are kept at atmospheric pressure and ambient temperature, thus any changes will affect the density of the gases and hence the refractive index. Using the above histogram it is possible to monitor changes in the refractivity $(n-1)$ of $0.1 \%$ or less.

\section{Calibration of particle ID performance}

In addition to the calibration methods presented above, we can evaluate the performance of the particle identification algorithms using data, independent of the LHCb Monte-Carlo simulation. This will be achieved using the $\mathrm{D}^{*}$ decay chain [10]:

$\mathrm{D}^{*+} \rightarrow \mathrm{D}^{0}+\pi^{+}, \quad \mathrm{D}^{0} \rightarrow \mathrm{K}^{-}+\pi^{+}$.

A dedicated trigger will select up to $10^{7} \mathrm{D}^{*}$ 's for $2 \mathrm{fb}^{-1}$ of data, independent of the RICH.

A high purity $\mathrm{D}^{*+}$ sample (above $90 \%$ ) can be selected through tight cuts on the $\mathrm{D}^{*+}-\mathrm{D}^{0}$ mass difference and B-decay impact parameter requirements. It is possible to further improve the purity of the sample by using electromagnetic calorimeter and muon detector information to reject the lepton contamination. In addition, the $\chi^{2}$ of the fitted track for the slow pion will be used to reject fake ("ghost") tracks.

Due to the tight kinematical constraint on the $\mathrm{D}^{*+}-\mathrm{D}^{0}$ mass difference, having a pure sample of $\mathrm{D}^{*}$ 's also gives a pure sample of pions and kaons from the $\mathrm{D}^{0}$ decay, since the kinematics of the decay are used to assign the particle masses. The particle identification performance of the RICH system can then be evaluated by comparing the pion and kaon assignments from the $\mathrm{RICH}$ reconstruction algorithm with the known pion and kaon selections from the $\mathrm{D}^{*}$ decays. Fig. 5 shows the performance of the RICH using this method (i.e. without recourse to Monte-Carlo information) compared with the performance evaluation using Monte-Carlo truth. Since background pions and kaons have different transverse momentum $\left(P_{\mathrm{t}}\right)$ distributions to those originating from the $\mathrm{D}^{*}$ chain (Eq. (2)), the calibration is performed in bins of both momentum and transverse momentum of the pions and kaons. In Fig. 5 a cut on $P_{\mathrm{t}}>1 \mathrm{GeV} / c$ has been applied on all pions and kaons and it can be seen that the $\mathrm{D}^{*}$ method can be used to reproduce the true performance of the LHCb RICH detectors extremely well. The performance of the RICH shown in Fig. 5 has been evaluated using simulated data from the

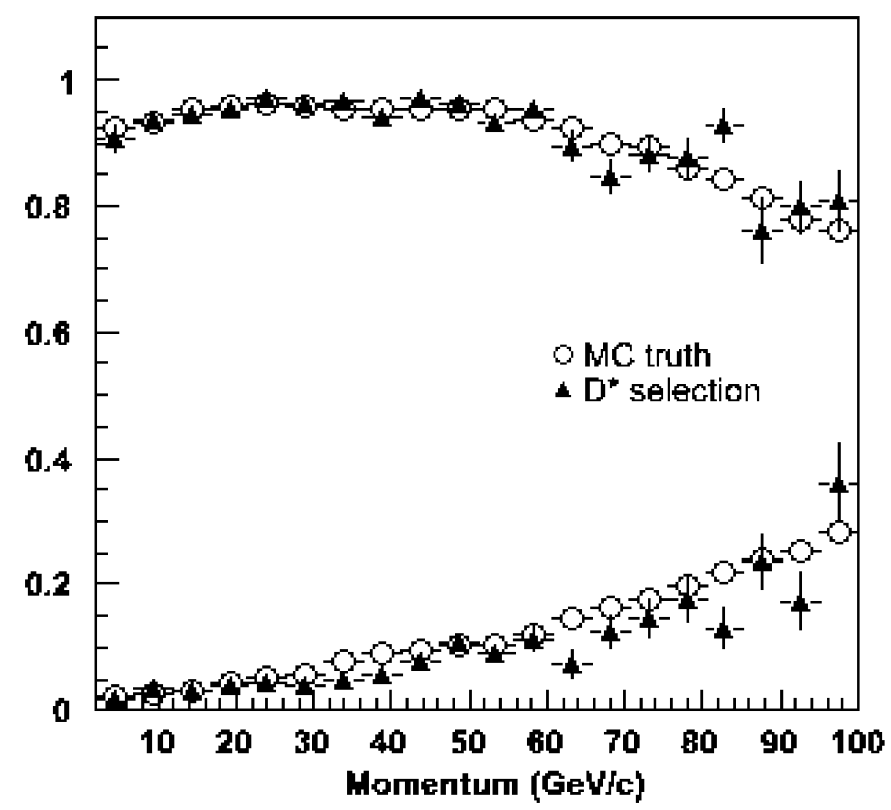

Fig. 5. RICH particle identification performance evaluated using purified samples of particles from the $\mathrm{D}^{*}$ decays defined in Eq. (2) (triangles), compared to using Monte-Carlo truth information from the LHCb simulation (open circles). The top curves show the efficiency in identifying kaons as heavy particles (kaons or protons) and the bottom curves show the misidentification of kaons as light particles (electrons, muons or pions). There is good agreement when a $P_{\mathrm{t}}>1 \mathrm{GeV}$ cut is applied on all pions and kaons. 
LHCb Data Challenge 2006 [11]. Recent improvements have pushed the average efficiency well above $90 \%$ while keeping the misidentification rate below $5 \%$.

\section{Summary}

The LHCb RICH system needs to be accurately calibrated and aligned to achieve maximum performance. The mirrors will be aligned to an accuracy of $0.1 \mathrm{mrad}$ and the refractivity $(n-1)$ monitored to an accuracy of $0.1 \%$. Correction methods for magnetic distortions in the HPD photon detectors are in the implementation phase. Cherenkov angle resolutions and photon yields will be calibrated using saturated Cherenkov rings and particles of known type. The performance of the RICH system will be evaluated using samples of pions and kaons identified from $\mathrm{D}^{*}$ decays, which can be isolated with high purity independent of the RICH itself.

\section{References}

[1] S. Amato, et al., the LHCb collaboration, LHCb RICH: Technical Design Report, CERN-LHCC-2000-037, 2000.

[2] S. Amato, et al., the LHCb collaboration, LHCb Technical Proposal, CERN-LHCC98-004, 1998.

[3] R. Antunes-Nobrega, et al., the LHCb collaboration, LHCb reoptimised detector design and performance, Technical Design Report 9, CERN-LHCC-2003-030, 2003.

4] T. Gys, Nucl. Instr. and Meth. A 567 (2006) 176.

[5] G. Aglieri Rinella, et al., Nucl. Instr. and Meth. A 553 (2005) 120.

[6] W. Baldini, et al., LHCb alignment strategy, CERN-LHCB-2006-035, 2006.

[7] A. Gorišek, et al., Nucl. Instr. and Meth. A 433 (1999) 408.

[8] S. Brisbane, et al., Nucl. Instr. and Meth. A 595 (2008) 146.

[9] S. Eisenhardt, Nucl. Instr. and Meth. A 595 (2008) 142

[10] R. Muresan, Nucl. Phys. Proc. Suppl. 170 (2007) 237.

[11] C. Buszello, et al., Nucl. Instr. and Meth. A 595 (2008) 245. 